\title{
Assessment of traffic noise due to transverse rumble strips at residential areas
}

\author{
Haron Zaiton ${ }^{1}$, Darus Nadirah ${ }^{1, *}$, Supandi Mohamad Afif ${ }^{1}$, Yahya Khairulzan $^{1}$, Mashros \\ Nordiana $^{2}$, and Jahya Zanariah ${ }^{1}$ \\ ${ }^{1}$ Department of Structure and Materials, Faculty of Civil Engineering, Universiti Teknologi Malaysia, \\ 81310 Johor Bahru, Johor, Malaysia \\ ${ }^{2}$ Department of Geotechnics and Transportation, Faculty of Civil Engineering, Universiti Teknologi \\ Malaysia, 81310 Johor Bahru, Johor, Malaysia
}

\begin{abstract}
Transverse rumble strips (TRS) are commonly being installed to alert the drivers through sound and vibration effects. The sound produced affects the existing traffic noise level which caused noise annoyance to the nearby residents. This study aims to assess the traffic noise due to TRS at residential areas by determining the roadside noise levels, traffic and road characteristics and evaluating the relationship between these parameters. Middle overlapped (MO), middle layer overlapped (MLO) and raised rumbler (RR) TRS profiles with same thickness were selected. The measurements of roadside noise levels and skid resistance were conducted using sound level meter (SLM) and British pendulum tester (BPT) respectively. Traffic characteristics were evaluated using previous data measured using automatic traffic counter (ATC). In overall, MLO produced highest roadside noise levels with increase of 20.5dBA from baseline. Generally, the increase of roadside noise level due to TRS is strong with speed, weak to medium with skid resistance of TRS and no relationship with traffic volume. Based on three TRS profile types, MLO is not suitable to be installed on the roadways adjacent to the residential areas as the increase of roadside noise level is significant which is more than $5 \mathrm{dBA}$ compared to MO and RR.
\end{abstract}

\section{Introduction}

Basically, the types of rumble strips are characterized by the position being installed on the roadways such as transverse, shoulder and centerline. In Malaysia, transverse rumble strips (TRS) are widely used as compared to shoulder (SRS) and centerline rumble strips (CRS) to alert the drivers on the upcoming hazards through sound and vibration effects $[1,2]$. TRS profile designs are uniform over the country according to the local authorities [3-5]. However, these guidelines are very basic which resulted in different TRS designs in term of dimension and profile. The most common TRS profile types are middle overlapped (MO), middle layer overlapped (MLO) and raised rumbler (RR) which generate different sound level can affect the residents lived adjacent to the roadways.

\footnotetext{
*Corresponding author: nddarus@yahoo.com
} 
Nowadays, living environment in many residential areas have been deteriorating due to the increase in traffic volume which resulted in noise annoyance to the nearby residents. The increases of noise level when the vehicle hit the TRS causes noise annoyance to the adjacent residents $[6,7]$. Noise complaints due to TRS were lodged by the affected residents to the local authorities [8-10]. Generally, noise pollution is dominantly caused by the traffic sources including engine power, frictional contact of vehicle and road surface, traffic volume, speed, composition and road surface condition [11, 12]. Road surface condition especially skid resitance is one of the TRS installation criteria set by the Public Work Department (PWD) with skid resistance value (SRV) of $\geq 45$ and $\geq 55$ for old and new TRS respectively [5].

Based on the previous research of roadside noise level, the application and effects of TRS installation on the roadway to the traffic noise climate condition have been investigated [6]. The results show that the TRS has increased the sound level indices $\mathrm{L}_{\text {Aeq, }}$, $\mathrm{L}_{10}, \mathrm{~L}_{90}$ significantly and there are changes in traffic noise index (TNI) which indicate dissatisfaction. Moreover, the installation of TRS has also increased the noise annoyance among the nearby residents. A $3 \mathrm{dBA}, 5 \mathrm{dBA}$ and $10 \mathrm{dBA}$ change in noise level is considered just discernible, clearly discernible and as a doubling or halving of volume respectively [13]. In Malaysia, the Department of Environment (DOE) has set the maximum permissible limit of 55dBA for 15hours daytime sound level at suburban residential areas [14].

Therefore, a clear view of the traffic noise due to TRS at residential is required to certainly help a better understanding on the actual environmental conditions. This will assists the local authorities in determining the appropriate design of TRS profile with acceptable noise level and good skid resistance value. This study intends to assess traffic noise due to TRS at residential areas in Johor where it was conducted accordingly based on the objectives of the study. The first objective is to determine the roadside noise levels, traffic characteristics and road characteristic. Meanwhile, the second objective is to evaluate the relationship between roadside noise levels with traffic characteristics. The last objective is to analyse the relationship between roadside noise levels with road characteristic specifically skid resistance.

\section{Methodology}

This study was conducted in three main stages including the determination of roadside noise levels specifically equivalent continuous sound pressure level $\left(\mathrm{L}_{\mathrm{Aeq}}\right)$, evaluation of traffic characteristics including volume, speed and composition and evaluation of road characteristic specifically skid resistance. The details of each stage were described in the following paragraphs.

\subsection{Determination of roadside noise levels}

The selection of measurement locations and TRS samples were based on the types of TRS profiles and location of TRS installation. Table 1 shows three different types of TRS profiles with same thickness of $3 \mathrm{~mm}$ selected for this study in order to compare their effect in terms of roadside noise levels on adjacent residents. The first experimental sites was located at Johor Bahru Tengah Municipal Council area which is at Sri Skudai with multilayer overlapped (MLO) profile installed in front of the shops and mosque. The second site is at Kangkar Pulai also under the same authority with middle overlapped (MO) profile installed in front of shops and residential houses. The third location is at Parit Karjo under Batu Pahat Municipal Council with raised rumbler (RR) profile installed along the roadways adjacent to the residential areas. 
Table 1. TRS samples.

\begin{tabular}{|c|c|c|c|}
\hline Types of profile & $\begin{array}{c}\text { Middle overlapped } \\
\text { (MO) }\end{array}$ & $\begin{array}{c}\text { Middle Layer } \\
\text { Overlapped (MLO) }\end{array}$ & $\begin{array}{c}\text { Raised Rumbler } \\
\text { (RR) }\end{array}$ \\
\hline Dimensions & $\rightarrow(300 m n$ & $\stackrel{+}{1} \longleftarrow$ & 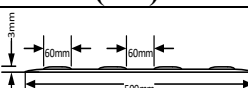 \\
\hline Thickness & $3 \mathrm{~mm}$ & $3 \mathrm{~mm}$ & $3 \mathrm{~mm}$ \\
\hline Width & $600 \mathrm{~mm}$ & $400 \mathrm{~mm}$ & $500 \mathrm{~mm}$ \\
\hline Spacing & $2.35 \mathrm{~m}$ & $2.45 \mathrm{~m}$ & $2.50 \mathrm{~m}$ \\
\hline Length of strip & $3.35 \mathrm{~m}$ & $2.80 \mathrm{~m}$ & $3.00 \mathrm{~m}$ \\
\hline Number of strips & 33 & 30 & 20 \\
\hline
\end{tabular}

Pulsar Type 1 Model 33 sound level meter (SLM) as shown in Fig. 1 was used to determine equivalent continuous sound pressure level $\left(\mathrm{L}_{\text {Aeq }}\right)$ values. As in Fig. 2, the measurements were conducted at the roadside with TRS (Position 1) and without TRS (Position 2). Wind speed and air temperature were recorded for every half an hour in order to ensure the values are below $5 \mathrm{~m} / \mathrm{s}$ and $5 \mathrm{C}$ to $40 \mathrm{C}$ for wind speed and air temperature respectively [15]. The measurement was conducted for 15 hours $(7.00$ a.m. to 10.00 p.m.) as stated by the Department of Environment, Malaysia [14]. The roadside noise levels due to actual traffic characteristics were measured at the roadways with and without TRS for three days within the middle of the week which include Monday, Tuesday, and Wednesday.

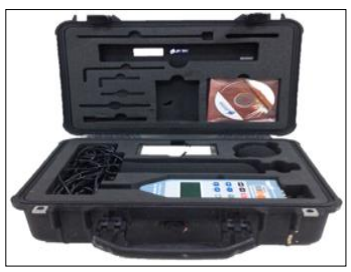

Fig. 1. Sound level meter.

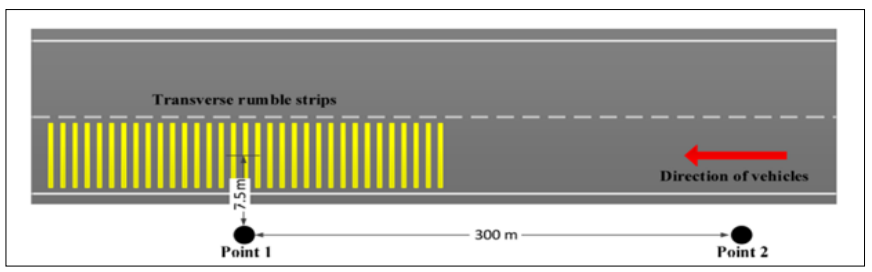

Fig. 2. Measurement layout.

\subsection{Evaluation of traffic characteristics}

Traffic characteristics including traffic volume, speed and composition each selected site was measured using automatic traffic counter (ATC). The measurements also were conducted for three days within the middle of the week which include Monday, Tuesday, and Wednesday which are the same with measurements of roadside noise levels. The start and end days of the week were excluded for to avoid different traffic behaviour at the measurement locations. The traffic volume and speed were measured in the units of $\mathrm{veh} /$ hour and $\mathrm{km} / \mathrm{h}$ respectively. ATC consists of a data recorder and two pneumatic road tubes installed parallel across the roadways. The vehicle passed the pneumatic road tube laid across the roadway and the data were transferred to the data recorder.

The additional sound may produce when the vehicle passes through the pneumatic road tube. Therefore, roadside noise levels were measured separately with the traffic characteristics measurement during the same days of different weeks. The previous traffic characteristics data were extracted to be used in this study. The influence of traffic charateristics on roadside noise level due to TRS were analysed by using simple linear regression with $\mathrm{R}^{2}$ determination to establish relationship. The relationships of each traffic characteristic on roadside noise levels were established. According to previous research, a rough rule of thumb for $\mathrm{R}^{2}$ of $0.25,0.50$ and 0.75 are weak, moderate and substantial respectively [16]. 


\subsection{Evaluation of road characteristic}

Road characteristic specifically skid resistance was evaluated using British pendulum tester (BPT) in accordance with the British Standards for Roads and Airfields (BS EN 13036:1 2003) [17]. BPT was operated by releasing a pendulum from a height that is adjusted so that a rubber slider on the pendulum head contacts the pavement surface over a fixed length. The measurement was conducted both in dry and wet conditions of road and TRS surfaces. The surface temperature was within $\left(25 \pm 2^{\circ} \mathrm{C}\right)$ during the measurement to prevent the effect of temperature on the BPT results. Similarly, the influence of skid resistance of TRS and increase of noise level due to TRS was also analysed by using simple linear regression with $\mathrm{R}^{2}$ determination.

\section{Results and discussions}

The data obtained from the on-site measurements and previous data were discussed and analysed in order to determine the roadside noise levels, traffic characteristics and road characteristic. Then, the relationships between the roadside noise levels with traffic characteristics and road characteristic were established.

\subsection{Roadside noise level}

Fig. 3, Fig. 4 and Fig. 5 show the roadside noise level differences between the roadways with and without TRS at three measurement sites namely Kangkar Pulai (MO), Sri Skudai (MLO) and Parit Karjo (RR) respectively. Based on these figures, the difference of roadside noise levels installed with and without MO and RR do not give a significant difference. Meanwhile, the difference of roadside noise levels installed with and without MLO shows a slight difference. All sites exceeded maximum permissible sound level (LAeq) of 55dBA. Fig. 6 shows that the difference of the noise level at Sri Skudai (MLO) was higher than site Parit Karjo (RR) and Kangkar Pulai (MO). The highest different was 20.5dBA at Sri Skudai (MLO).

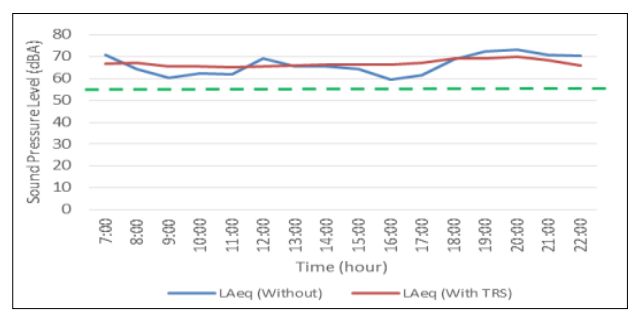

Fig. 3. Kangkar Pulai (MO).

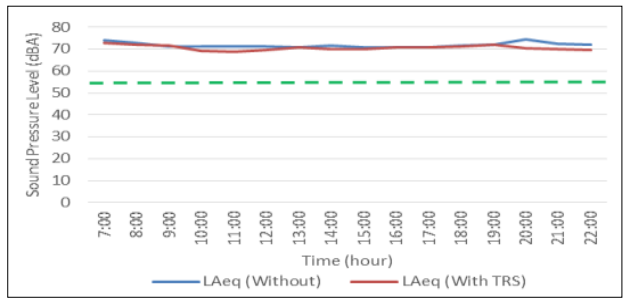

Fig. 5. Parit Karjo (RR).

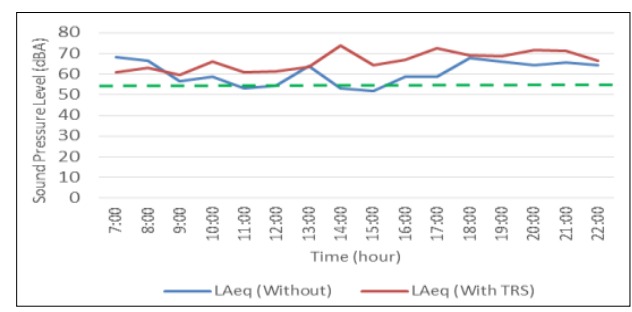

Fig. 4. Sri Skudai (MLO).

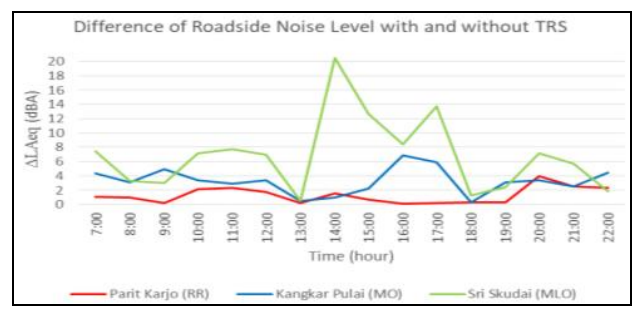

Fig. 6. Difference of roadside noise levels. 


\subsection{Traffic characteristics}

\subsubsection{Traffic volume}

The study only focused on the middle of the week which are Monday, Tuesday, and Wednesday. Based on the previous data, the traffic characteristics pattern within these days is similar at each measurement locations. Therefore, the measurements were conducted during these three days for all locations. The measurement was conducted on Tuesday at Kangkar Pulai (MO) and Sri Skudai (MLO). Meanwhile, the measurement was conducted on Wednesday at Parit Karjo (RR). Figure 7 shows the traffic volume for all sites at the selected day for the roadside noise level measurement. The traffic volume at the Kangkar Pulai (MO) shows the highest daily traffic volume as compared to Kangkar Pulai (MO) and Sri Skudai (MLO).

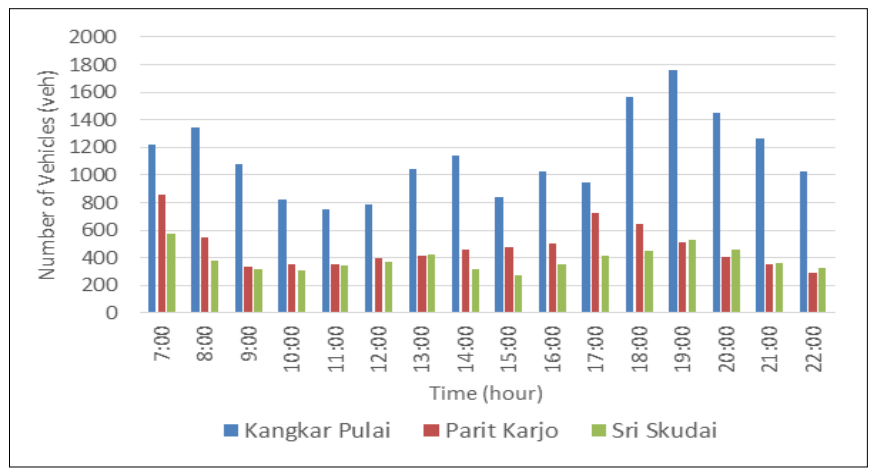

Fig. 7. Traffic volume.

\subsubsection{Traffic speed}

Figure 8 shows the average traffic speed for all measurement locations. The data showed that the average speed at Parit Karjo (RR) is higher than Kangkar Pulai (MO) and Sri Skudai (MLO) which is around 65 to $72 \mathrm{~km} / \mathrm{h}$. Meanwhile, the average speed of Kangkar Pulai and Sri Skudai are 45 to $50 \mathrm{~km} / \mathrm{h}$ and 30 to $55 \mathrm{~km} / \mathrm{h}$ respectively. The posted speed limit at all sites are $60 \mathrm{~km} / \mathrm{h}$ where only average speed at Parit Karjo (RR) exceeded the limit.

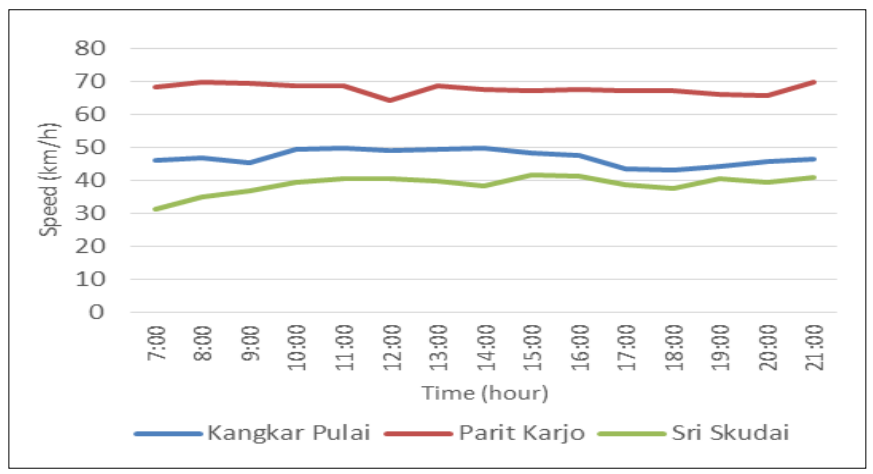

Fig. 8. Average traffic speed. 


\subsubsection{Traffic composition}

Based on Figure 9, Class 2 vehicles have the highest number of daily vehicles as compared to other classes of vehicle. Class 2 includes all passenger cars. Meanwhile, the second highest is Class 1 vehicles which include motorcycles, motor scooters, motor-powered bicycles, and three-wheeled motorcycles. Other class of vehicles are Class 3: other twoaxle, four-tire, single unit vehicles, Class 4: buses, Class 5: two-axle, six-tire, single unit trucks, Class 6: three-axle single unit trucks, Class 7: four or more axle single unit trucks, Class 8: four or less axle single trailer trucks, Class 9: five-axle single trailer trucks, Class 10: six or more axle single trailer trucks, Class 11: five or less axle multi-trailer trucks, Class 12: six-axle multi-trailer trucks and Class 13: seven or more axle multi-trailer.

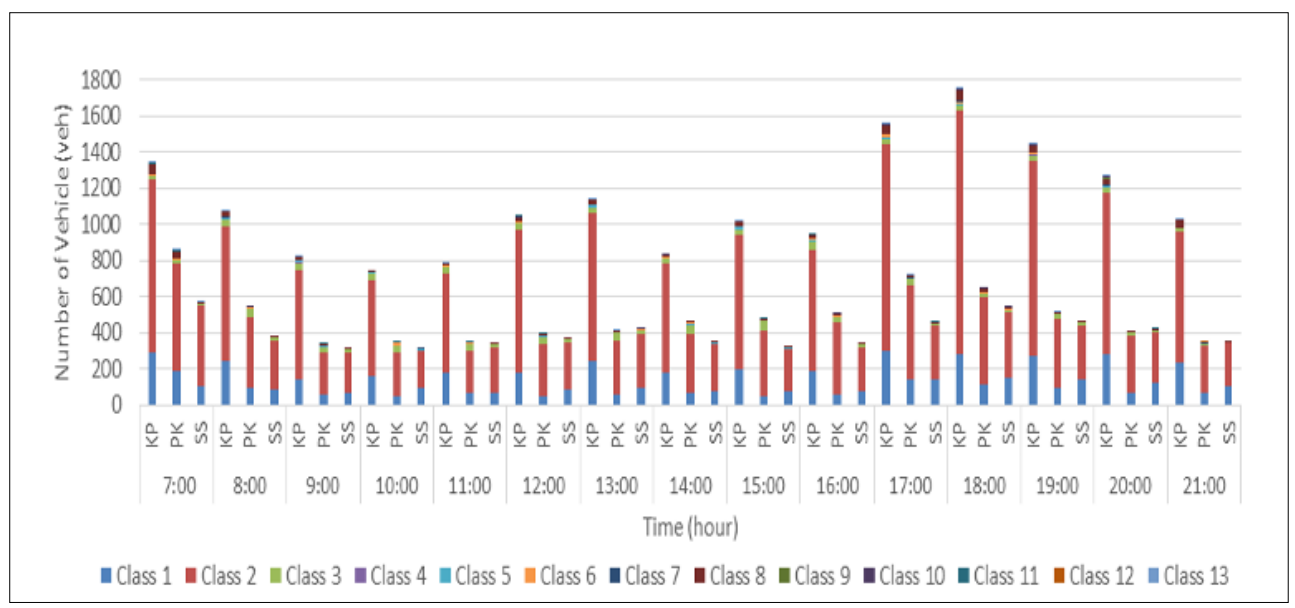

Fig. 9. Traffic composition.

\subsection{Road characteristic}

\subsubsection{Skid Resistance}

Table 2 shows the skid resistance value (SRV) at all sites with and without TRS. The SRV was compared with the criteria for TRS installation set by the Public Works Department (PWD). All TRS profile have passed the criteria limit of $\geq 45$ SRV for old or existing TRS in dry and wet conditions. The selected TRS profiles for this study are within acceptable SRV and in good condition.

Table 2. Skid resistance value (SRV).

\begin{tabular}{|c|c|c|c|c|c|}
\hline \multirow{2}{*}{ Measurement Sites } & \multicolumn{2}{|c|}{ Without TRS } & \multicolumn{2}{c|}{ With TRS } & \multirow{2}{*}{ SRV by PWD } \\
\cline { 2 - 5 } & Dry & Wet & Dry & Wet & \\
\hline Kangkar Pulai (MO) & 77 & 51 & 90.50 & 51.67 & \multirow{2}{*}{$\geq 45$} \\
\hline Sri Skudai (MLO) & 81 & 50 & 94.60 & 56.33 & \\
\hline Parit Karjo (RR) & 90 & 55 & 93.60 & 56.00 & \\
\hline
\end{tabular}




\subsection{Relationship between roadside noise level and traffic volume}

Figure 10 to Figure 13 show the relationship between noise levels and traffic volume. The roadside noise level is directly proportional to the traffic volume as can be observed in Figure 12 as compared to Figure 10 and Figure 11. Figure 13 shows that the average of different noise level not directly proportional to the average traffic volume (very low $\mathrm{R}^{2}=0.12$ ), because of the difference of noise level affected by the type of the TRS. MLO show the higher average difference noise level, but the traffic volume was lower than RR and MO. Based on previous study, the sound level changes for vehicles transit on MLO are higher than MO and RR because of the impulsivity and the patent of the TRS, while RR show lower difference roadside noise level because of the tonality [17].

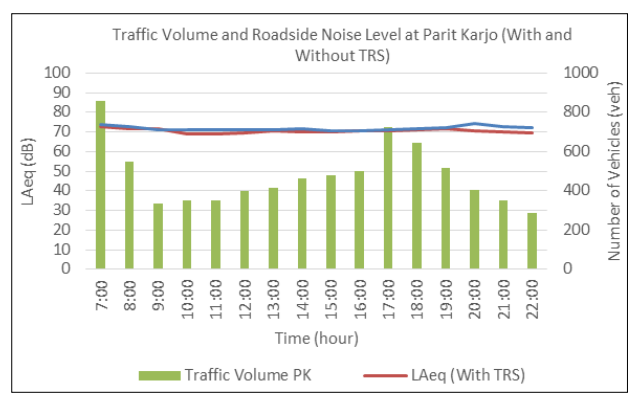

Fig. 10. Parit Karjo (RR).

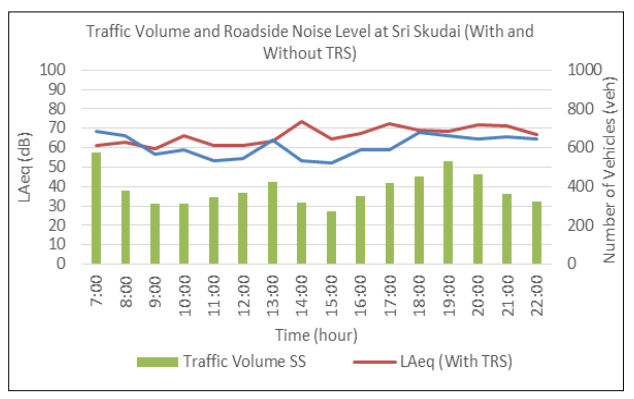

Fig. 12. Sri Skudai (MLO).

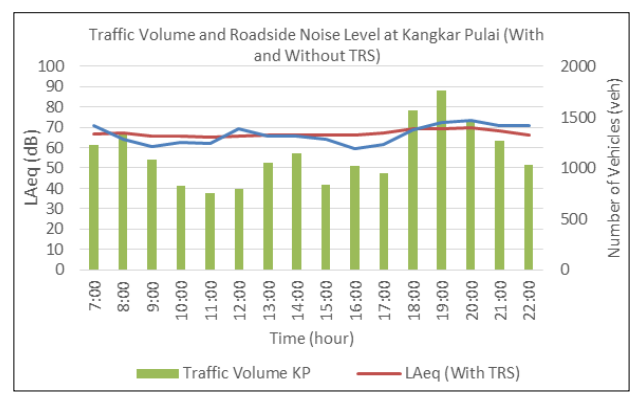

Fig. 11. Kangkar Pulai (MO).

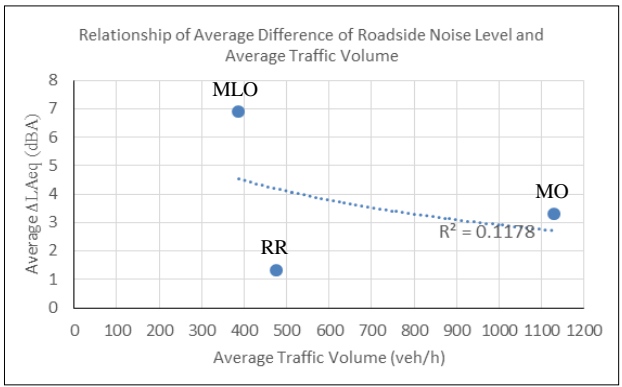

Fig. 13. Average difference of roadside noise level vs. average traffic volume.

\subsection{Relationship between roadside noise level and traffic speed}

Figure 14 to Figure 16 show that the relationship between noise level and traffic speed. The average speed between 30 to $50 \mathrm{~km} / \mathrm{h}$ produces the higher noise level than the speed more than $60 \mathrm{~km} / \mathrm{h}$. Compared with the previous study which stated that MO seemed to give more significant impulsive characteristics at low speed than MLO and shows the best performance for speed of $50 \mathrm{~km} / \mathrm{h}$ [7]. Roadway installed with MLO type of TRS produce the higher noise level different from the others type. Figure 17 shows the high relationship between the average difference of roadside noise level and the average speed $\left(\mathrm{R}^{2}=0.99\right)$. The average difference of noise level decrease when the average speed approaching the 70 $\mathrm{km} / \mathrm{h}$, the optimal speed which causes the minimum value of noise level [18]. Based on previous study, it is also stated that sound level changes for vehicles transit on MLO are higher than MO and RR [19]. 


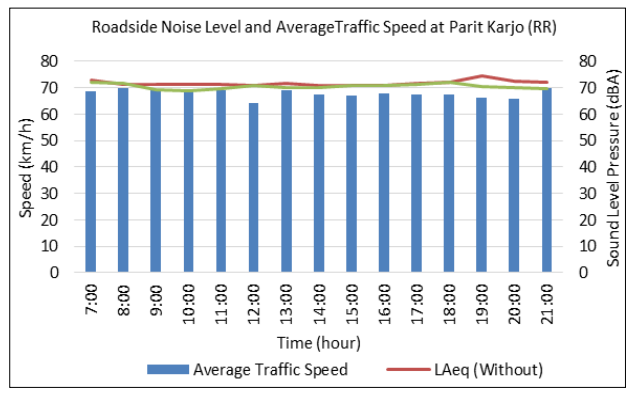

Fig. 14. Parit Karjo (RR).

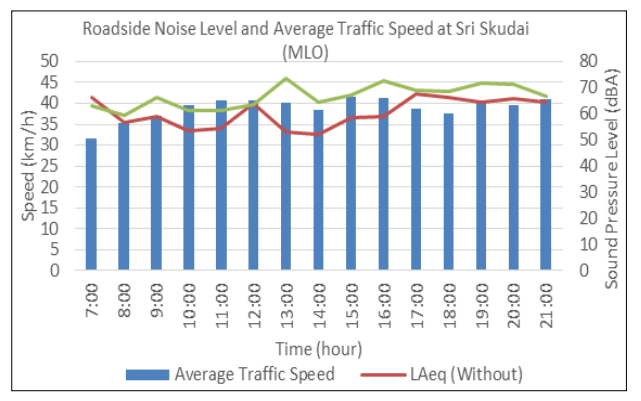

Fig. 16. Sri Skudai (MLO).

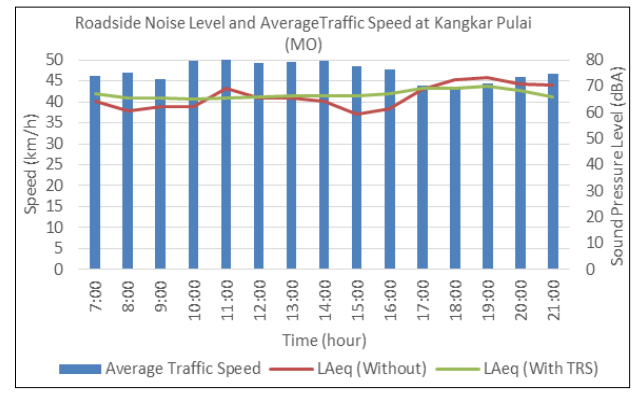

Fig. 15. Kangkar Pulai (MO).

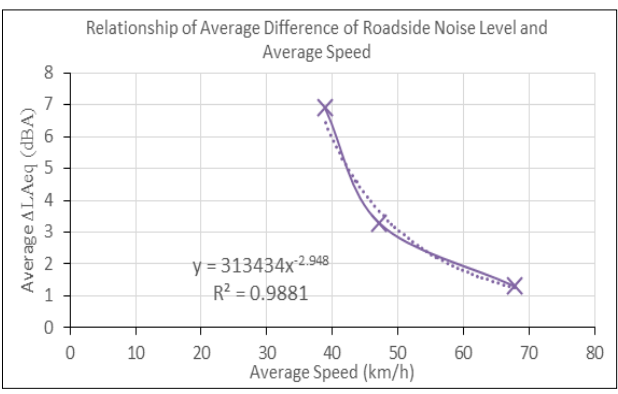

Fig. 17. Average difference of roadside noise level vs. average traffic speed (with and without TRS).

\subsection{Relationship between roadside noise level and skid resistance}

Figure 18 shows that the relationship between the average difference of roadside noise level and skid resistance. It resulted as weak to medium influence on increase of roadside noise level $\left(\mathrm{R}^{2}=0.36\right)$. Although the thickness are same, the type of the TRS gives the difference in noise level. Roadside noise level changes due to the characteristics of the TRS such as depth and type of TRS.

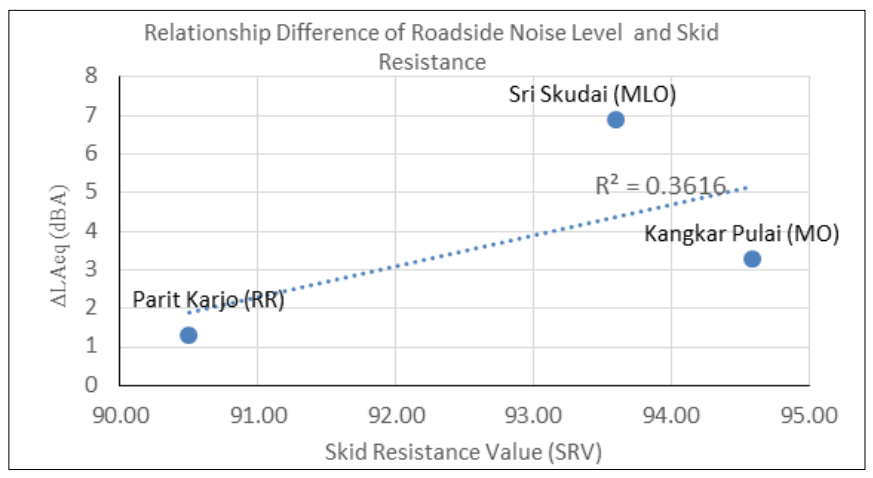

Fig. 18. Average difference of roadside noise level vs. Skid resistance value. 


\section{Conclusions}

Assessment of the traffic noise due to transverse rumble strip (TRS) installed at residential areas were carried out in this study. Overall, it can be concluded that the roadside noise level of roadway installed with TRS is higher than the roadway without TRS. Regarding the types of TRS profile, MLO produced higher roadside noise levels as compared to MO and RR. The highest difference of roadside noise level installed with MLO was 20.5dBA. In terms of traffic characteristics, Parit Karjo (RR) recorded highest traffic volume and average speed as compared to the other two sites installed with MO and MLO. Meanwhile, Class 2 vehicles including all passenger cars are the highest traffic composition followed by Class 1 at all sites.

Skid resistance of road and TRS surfaces at all three measurement sites are within the limit of $\geq 45 \mathrm{SRV}$. For the different TRS profiles with the same thickness, it can be summarized that the relationship between the roadside noise level and traffic volume is not directly proportional, there is strong relationship between the roadside noise level and traffic speed and there is weak to medium relationship between the roadside noise level and skid resistance of TRS. Based on the three TRS profile types, MLO is not suitable to be installed on the roadways near to the residential areas because MLO produced a significant increase of roadside noise level which is more than $5 \mathrm{dBA}$ as compared to $\mathrm{MO}$ and RR. It may affect the nearby residents in terms of traffic noise annoyance.

The study was funded by GUP Q.J130000.2522.15H28, GUP Q.J130000.2522.19H76, FRGS R.J130000.7822.4F887 and R.J130000.7822.4F867 which supported by Research Management Centre, Universiti Teknologi Malaysia. These supports are highly appreciated.

\section{References}

1. G. Bahar, T. Erwin, M. Mackay, A. Smiley, S. Tighe, Best practice guidelines for the design and application of transverse rumble strips (Trans. Assoc. of Canada, Ottawa, Canada, 2005)

2. Y. Yang, X. Sun, Y. He, Effectiveness of rumble strips on freeways (A paper presented ICCTP 2010: Integrated Transportation Systems, 425-433, 2010)

3. Ministry of Work (MOW), Traffic calming guidelines (Highway Planning Unit, Kuala Lumpur, 2002)

4. Road Engineering Association of Malaysia (REAM), Guidelines on traffic control and management devices (REAM, Kuala Lumpur, 2004)

5. Public Work Department (PWD), Manual fasiliti keselamatan jalan (Cawangan Kejuruteraan Jalan \& Geoteknik, Malaysia, 2014)

6. Z. Haron, M. H. Othman, K. Yahya, H. Yaacob, M. R. Hainin, M. B. Mohd Yusof, J. of Mech. \& Civil Eng. Noise produced by transverse rumble strips: a case study on rural roadways, 1(5), 12-16 (2012)

7. Z. Haron, M. H. Othman, M. H. Lim, K. Yahya, H. Yaacob, M. R. Hainin, N. Darus, M. S. Leong, Exterior noise due to interaction of tyre-thermoplastic transverse rumble strips, Archives of Acoustics, 42(3) (2017)

8. T. K. Datta, T. J. Gates, P. T. Savolainen, Impact of non-freeway rumble strips phase 1 (ORBP reference number: OR09084A, 2012)

9. M. H. Othman, Performance and modelling of transverse rumble strips on noise and vibrations stimuli, PhD thesis, (Universiti Teknologi Malaysia, Malaysia, 2016)

10. J. S. Higgins, W. Barbel, J. of the Trans. Research Board, Rumble strip noise, 983, 27 (1984) 
11. A. A. Kadar Hamsa, M. Miura, S. Inokuma, Y. Nishimura, J. of Asian Arch. and Build. Eng. Evaluating the living environment in residential areas at Taman Melati, Kuala Lumpur, 5(2), 377 (2006)

12. M. J. Damavandi, M. Nowrouzi, Energy and Env.: Recent Researches in Env. and Geo. Sciences, Analysis of noise nuisance on residents of the Malaysian condominiums: heritage condominium case study, 266 (2012)

13. J. L. Darbyshire, J. D. Young, Critical Care, An investigation of sound levels on intensive care units with reference to the WHO guidelines, 17(187), (2013)

14. Department of Environment (DOE), The planning guidelines for environmental noise limits and control (Ministry of Natural Resources and Environment, Malaysia, 2004)

15. International Organization of Standardization (ISO), ISO 11819-1: 2001, Acoustics measurement of the influence of road surfaces on traffic noise - part 1: statistical passby method (Brussels: European Committee for Standardization, 2001)

16. W. W. Low, H. Abdul Rahman, N. Zakaria, Int. J. of Project Mngmnt, The impact of organizational culture on international bidding decisions: Malaysia context, 33, 917931 (2015)

17. British Standard Instituition (BSI), BS-EN 13036-4: 2003, Road and airfield surface characteristics, part 4: method for measurement of slip/skid resistance of a surface: the pendulum test (Technical Committee CEN/TC 227 "Road Materials", 2003)

18. M. Miraglia, Discrete and incoherent linear sources, Lezione del, Retrieved from pcfarina.eng.unipr.it/Public/Acoustics-Course/DOC-2012/07_miraglia_221916.doc (2012, October 19)

19. N. Darus, Z. Haron, K. Yahya, M. H. Abd Halil, W. M. A. Norudin, M. H. Othman, M. A. Hezmi, E3S Web Conference, Impulsivity of noise due to single vehicles transit on transverse rumble strips, 34, 02026 (2018) 